\title{
Exact multiplicity of positive solutions for the one- dimensional Minkowski-curvature problem with convex-concave nonlinearity
}

Hongliang Gao ( $\square$ gaohongliang101@163.com )

Lanzhou Jiaotong University https://orcid.org/0000-0003-1318-8551

Jing Xu

Lanzhou Jiaotong University

\section{Research Article}

Keywords: Minkowski-curvature equation, Exact multiplicity, Positive solution, Bifurcation curves, Time map

Posted Date: January 25th, 2022

DOI: https://doi.org/10.21203/rs.3.rs-1063439/v1

License: (c) (1) This work is licensed under a Creative Commons Attribution 4.0 International License.

Read Full License 


\title{
Exact multiplicity of positive solutions for the one-dimensional Minkowski-curvature problem with convex-concave nonlinearity
}

\author{
Hongliang Gao Jing Xu \\ Department of Mathematics, LanZhou Jiaotong University, Lanzhou 730070, P R China
}

Abstract. We establish the bifurcation curves and exact multiplicity of positive solutions for the following problem with mean curvature operator in the Minkowski space

$$
\left\{\begin{array}{l}
-\left(\frac{u^{\prime}}{\sqrt{1-u^{\prime 2}}}\right)^{\prime}=\lambda f(u), \quad x \in(-L, L), \\
u(-L)=0=u(L),
\end{array}\right.
$$

where $\lambda$ and $L$ are positive parameters, $f \in C^{1}[0, \infty) \cap C^{2}(0, \infty)$ satisfies $f(0)=0$ and $f(u)>0$ for $u \in(0, L)$, and $f$ is convex-concave on $(0, L)$. By a detailed analysis of the time map, we determine the interval of parameter $\lambda$ in which the above problem has zero, exactly one or exactly two positive solutions when $f$ satisfies certain conditions.

Keywords. Minkowski-curvature equation; Exact multiplicity; Positive solution; Bifurcation curves; Time map

\section{MSC: 35J93, 34B18}

\section{Introduction}

The aim of this paper is to study the bifurcation curves and exact multiplicity of positive solutions to the following problem

$$
\left\{\begin{array}{l}
-\left(\frac{u^{\prime}}{\sqrt{1-u^{\prime 2}}}\right)^{\prime}=\lambda f(u), \quad-L<x<L \\
u(-L)=0=u(L)
\end{array}\right.
$$

where $\lambda$ and $L$ are positive parameters, $f \in C^{1}[0, \infty) \cap C^{2}(0, \infty)$ satisfies $f(0)=0$ and $f(u)>0$ for $u \in(0, L)$, and $f$ is convex-concave on $(0, L)$. In fact, problem (1.1) is one-dimensional version of the Minkowski-curvature equation with Dirichlet boundary value condition

$$
\left\{\begin{array}{l}
-\operatorname{div}\left(\frac{\nabla u}{\sqrt{1-|\nabla u|^{2}}}\right)=\lambda f(|x|, u), \quad x \in \Omega, \\
u=0, \quad x \in \partial \Omega
\end{array}\right.
$$

which is originated from differential geometry or classical relativity.

${ }^{*}$ Correspondence: gaohongliang101@163.com 
There are a large amount of papers in the literature on the existence of positive solutions for this type of problems (see e.g.[1-4], [6], [7], [11], [12] and the references therein). In particular, Coelho et al. [6] proved the existence and multiplicity of positive solutions for one-dimensional Minkowski-curvature equation

$$
\left\{\begin{array}{l}
-\left(\frac{u^{\prime}}{\sqrt{1-u^{\prime 2}}}\right)^{\prime}=\lambda f(t, u), \quad t \in(0, T), \\
u(0)=u(T)=0
\end{array}\right.
$$

under the assumption that $f$ is $L^{p}$-Carathéodory function and not required to be positive. Recently, using Leray-Schauder degree argument and critical point theory, the authors of [1] obtained some existence results for positive radial solutions of problem (1.2) with $\lambda=1$ and $\Omega=\left\{x \in \mathbb{R}^{N}|| x \mid<R\right\}$ for some constant $R>0, f: C([0, R] \times[0, \alpha), \mathbb{R}), 0<\alpha \leq \infty$ and $f(r, s)>0,(r, s) \in[0, R] \times[0, \alpha)$. In [2], they also established some nonexistence, existence and multiplicity results for positive radial solutions of problem (1.2) with $f(|x|, u)=\mu(|x|) u^{p}$, where $p>1$ and $\mu:[0, \infty) \rightarrow \mathbb{R}$ is continuous, strictly positive on $(0, \infty)$. Namely, there exists $\Lambda>0$ such that problem (1.2) has zero, at least one or at least two positive radial solutions according to $\lambda \in(0, \Lambda), \lambda=\Lambda$ or $\lambda>\Lambda$. Ma et al. [11] and Dai [3] also proved the nonexistence, existence and multiplicity of radial positive solution of problem (1.2) via bifurcation technique corresponding to asymptotically linear, sublinear, and superlinear nonlinearities $f$ at zero respectively, which generalized and improved the results of the literature $[1,2]$.

Nevertheless, it is worth noting that the above references mainly studied the existence but not the exactness of positive solutions. Recently, the exact number of the positive solutions of problem (1.1) has been considered by Zhang and Feng [15], Huang [13, 14], and Gao and Xu [5] under $u f^{\prime}(u) \leq f(u)$ and $f^{\prime \prime}(u)$ is not sign-changing. By a detailed analysis of the time map, they obtained some interesting results, which are quite different from the corresponding semilinear problem. To the best of our knowledge, there are no references in studying the exact multiplicity of positive solutions of Minkowski-curvature problem (1.1) if $f(u)$ is convex-concave function. Therefore, studying the exact multiplicity of positive solutions of Minkowski-curvature problem (1.1) is interesting and essential.

On the other hand, we noticed that the exact number of positive solutions for the corre- 
sponding semilinear problem of quasilinear problem (1.1)

$$
\left\{\begin{array}{l}
-u^{\prime \prime}(x)=\lambda f(u), \quad-1<x<1, \\
u(-1)=0=u(1)
\end{array}\right.
$$

has been largely studied by many scholars, where $\lambda$ is a positive parameter(see e.g.[8-10] and the references therein). In [9], Korman and Li proved the bifurcation curve of problem (1.3) is $S$-shaped if $\theta(\gamma)=2 \int_{0}^{\gamma} f(t) d t-\gamma f(\gamma) \leq 0, f \in C^{2}[0, \infty)$ satisfies $f(u)>0$ for $u \geq 0$ and $f$ is convex-concave on $(0, \infty)$ which implies $f^{\prime \prime}(u) \geq 0$ for $u \in(0, \gamma]$ and $f^{\prime \prime}(u)<0$ for $u \in(\gamma, \infty)$, and asymptotically sublinear at infty. Hung and Wang[8] improved the results in [9], since they weaken $\theta(\gamma) \leq 0$ as $H(\gamma)=3 \int_{0}^{\gamma} t f(t) d t-\gamma^{2} f(\gamma) \leq 0$. In [10], Korman and Li proved the bifurcation curve of problem (1.3) is $S$-shaped or reversed $S$-shaped when $f$ is convex-concave or concave-convex on $(0, \infty)$ and satisfies certain conditions.

Motivated by above papers, in this paper, we intend to study the bifurcation curves and exact multiplicity of positive solutions of problem (1.1). Now, we state the following hypothesis on the nonlinearity $f$ :

(H1) $f \in C([0, \infty), \mathbb{R})$ and $f(u)>0$ for $0<u<L$;

(H2) $f \in C^{2}(0, \infty), f(0)=0$ and there exists a number $0<\gamma<L$ such that

$$
f^{\prime \prime}(u) \begin{cases}>0, & \text { on }(0, \gamma) \\ =0, & \text { when } u=\gamma \\ <0, & \text { on }(\gamma, L)\end{cases}
$$

(H3) $f \in C^{1}[0, \infty) \cap C^{2}(0, \infty)$ and there exists $p \in(\gamma, L)$ such that

$$
u f^{\prime}(u)-f(u) \begin{cases}>0, & \text { on }[0, p), \\ =0, & \text { when } u=p, \\ <0, & \text { on }(p, L),\end{cases}
$$

and $u f^{\prime}(u) \geq f(u)+\frac{1}{2} u^{2} f^{\prime \prime}(u)$ for $u \in(0, p)$.

We consider the following two cases.

(C1) $\lim _{r \rightarrow 0^{+}} \frac{f(r)}{r}=A \in(0,+\infty)$ and $\lim _{r \rightarrow 0^{+}} f^{\prime \prime}(u) \in(0, \infty]$.

(C2) $\lim _{r \rightarrow 0^{+}} \frac{f(r)}{r^{\beta}}=A \in(0,+\infty), \beta>1$.

Our main results are as follows:

Theorem 1.1. Assume that $f$ satisfies (H1)-(H3) and (C1), then there exists $0<\lambda_{*}<\lambda^{*}=$ $\frac{\pi^{2}}{4 L^{2} A}$ such that 
(i) As $0<\lambda<\lambda_{*}$, problem (1.1) has no positive solution;

(ii) As $\lambda=\lambda_{*}$ and $\lambda \geq \lambda^{*}$, problem (1.1) has exactly one positive solution;

(iii) As $\lambda_{*}<\lambda<\lambda^{*}$, problem (1.1) has exactly two positive solutions. (see Fig.1(a))

Theorem 1.2. Assume that $f$ satisfies (H1)-(H3) and (C2), then there exists $\lambda_{*}>0$ such that

(i) As $0<\lambda<\lambda_{*}$, problem (1.1) has no positive solution;

(ii) As $\lambda=\lambda_{*}$, problem (1.1) has exactly one positive solution;

(iii) As $\lambda>\lambda_{*}$, problem (1.1) has exactly two positive solutions. (see Fig.1(b))

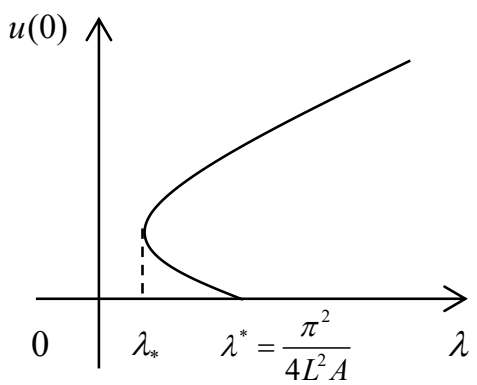

(a)

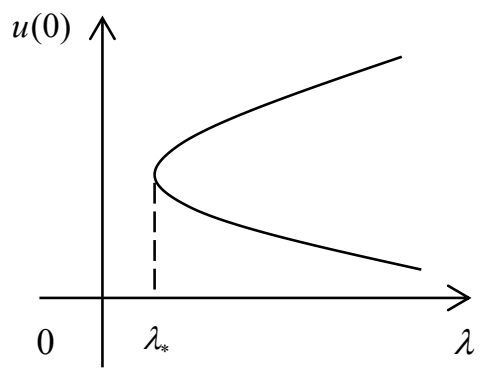

(b)

Fig. 1. (a) Graph of bifurcation curve of Theorem 1.1; (b) Graph of bifurcation curve of Theorem 1.2. Remark 1.3. It follows from $\lim _{r \rightarrow 0^{+}} \frac{f(r)}{r^{\beta}}=A \in(0,+\infty), 0<\beta<1$ that $\lim _{r \rightarrow 0^{+}} \frac{f(r)}{r}=+\infty$. However, (H3) implies $\frac{f(u)}{u}$ is monotonically increasing on $(0, p)$. Therefore, $\beta \geq 1$ if (H3) holds.

Remark 1.4. If $f \in C^{3}((0, \infty), \mathbb{R})$, then $\lim _{r \rightarrow 0^{+}} \frac{f(r)}{r}=A \in(0,+\infty)$ and the condition (H3) imply that $f^{\prime \prime \prime}(r) \leq 0$ for small positive numbers $r$. However, for small positive numbers $r$, it is easy to obtain that $f^{\prime \prime}(r) \leq 0$ from $\lim _{r \rightarrow 0^{+}} f^{\prime \prime}(u)=0$ and $f^{\prime \prime \prime}(r) \leq 0$, which is a contradiction with $f^{\prime \prime}(r)>0$ for $0<u<\gamma$. Therefore, the case of $\lim _{r \rightarrow 0^{+}} \frac{f(r)}{r}=A \in(0,+\infty)$ and $\lim _{r \rightarrow 0^{+}} f^{\prime \prime}(u)=0$ may not occur if $f$ satisfies (H3).

The paper is organized as follows. In Section 2 we introduce and analyze the time map which plays a key role in this paper, and then we obtain some inequalities about the time map. Section 3 contains the proofs of the main results. In Section 4 some examples are presented to illustrate the main results.

\section{Preliminary lemmas}

In this section, we shall make a detailed analysis of the so-called time map for problem (1.1). Various properties of the time map will be used to obtain exact multiplicity of positive solutions for problem (1.1) with convex-concave nonlinearity. 
Assume that $f$ satisfies (H1). Let $u(x)$ be a positive solution of problem (1.1). It is well known that $u(x)$ takes its maximum at $x=0, u(x)$ is symmetric with respect to $0, u^{\prime}(x)>0$ for $-L \leq x<0$ and $u^{\prime}(x)<0$ for $0<x \leq L$. Therefore, problem (1.1) is equivalent to the following problem

$$
\left\{\begin{array}{l}
-\left(\frac{u^{\prime}(x)}{\sqrt{1-u^{\prime 2}(x)}}\right)^{\prime}=\lambda f(u(x)), \quad 0<x<L, \\
u^{\prime}(0)=0=u(L) .
\end{array}\right.
$$

Denote $F_{\lambda}(u)=\lambda \int_{0}^{u} f(s) d s, u(0)=r$. Multiplying both sides of the equation in (2.1) by $u^{\prime}(x)$ and then integrating from 0 to $x$ leads to

$$
-\int_{0}^{x} \frac{u^{\prime}(x) u^{\prime \prime}(x)}{\left[1-u^{\prime}(x)\right]^{\frac{3}{2}}} d x=F_{\lambda}(u(x))-F_{\lambda}(r) .
$$

Combining with (2.1), it follows that

$$
\frac{1}{\sqrt{1-u^{\prime 2}(x)}}-1=F_{\lambda}(r)-F_{\lambda}(u(x)) .
$$

Therefore,

$$
-u^{\prime}(x)=\frac{\sqrt{\left[1+F_{\lambda}(r)-F_{\lambda}(u(x))\right]^{2}-1}}{1+F_{\lambda}(r)-F_{\lambda}(u(x))},
$$

i.e.

$$
-\frac{1+\lambda F(r)-\lambda F(u(x))}{\sqrt{[1+\lambda F(r)-\lambda F(u(x))]^{2}-1}} d u(x)=d x .
$$

Integrating (2.3) from 0 to $L$ leads to

$$
\int_{0}^{r} \frac{1+\lambda F(r)-\lambda F(u)}{\sqrt{[1+\lambda F(r)-\lambda F(u)]^{2}-1}} d u=L .
$$

Let

$$
T_{\lambda}(r)=\int_{0}^{r} \frac{1+\lambda F(r)-\lambda F(u)}{\sqrt{[1+\lambda F(r)-\lambda F(u)]^{2}-1}} d u,
$$

Then the function $T_{\lambda}(r)$ is called the time map of $f$.

Notice that the positive solutions of (1.1) correspond to curves for which $T_{\lambda}(r)=L$. In fact, (2.2) implies $\left|u^{\prime}(x)\right|<1$. For a detailed discussion, we also see step 1 of Theorem 2.1 in [6]. Hence, $u(0)=r<L$.

For the sake of simplicity [15], in what follows, we usually denote $T_{\lambda}(r)$ by $T(r), \frac{\partial T}{\partial r}$ by $T^{\prime}$, and $\xi=\lambda(F(r)-F(r s)), \xi^{\prime}=\frac{\partial \xi}{\partial r}=\lambda(f(r)-s f(r s)), \xi^{\prime \prime}=\frac{\partial^{2} \xi}{\partial r^{2}}=\lambda\left(f^{\prime}(r)-s^{2} f^{\prime}(r s)\right)$, then

$$
T(r)=r \int_{0}^{1} \frac{1+\xi}{\sqrt{\xi(2+\xi)}} d s .
$$


Lemma 2.1.[15] Assume that (H1) holds, then the time map $T(r)$ has continuous derivatives up to the second order with respect to $r$, and

$$
\begin{gathered}
T^{\prime}(r)=\int_{0}^{1} \frac{\xi(1+\xi)(2+\xi)-r \xi^{\prime}}{(\xi(2+\xi))^{\frac{3}{2}}} d s \\
=\frac{1}{r} \int_{0}^{r} \frac{\Delta F(1+\Delta F)(2+\Delta F)-\Delta \bar{f}}{(\Delta F(2+\Delta F))^{\frac{3}{2}}} d u . \\
T^{\prime \prime}(r)=\int_{0}^{1} \frac{-2 \xi^{\prime} \xi(2+\xi)-r \xi^{\prime \prime} \xi(2+\xi)+3 r \xi^{\prime 2}(1+\xi)}{(\xi(2+\xi))^{\frac{5}{2}}} d r \\
=\frac{1}{r^{2}} \int_{0}^{r} \frac{3 \Delta \bar{f}^{2}(1+\Delta F)-\Delta F(2+\Delta F)\left(2 \Delta \bar{f}+\Delta \bar{f}^{\prime}\right)}{(\Delta F(2+\Delta F))^{\frac{5}{2}}} d u .
\end{gathered}
$$

where $\Delta F=F_{\lambda}(r)-F_{\lambda}(u), \Delta \bar{f}=r f_{\lambda}(r)-u f_{\lambda}(u), \Delta \bar{f}^{\prime}=r^{2} f_{\lambda}^{\prime}(r)-u^{2} f_{\lambda}^{\prime}(u)$.

Lemma 2.2.[15] Assume that (H1) holds, then for any $r \in(0, L)$, the time map $T$ is strictly decreasing with respect to $\lambda$.

Lemma 2.3.[15] Assume that (H1) holds, then

(1) If $f(0)>0$, then $\lim _{r \rightarrow 0^{+}} T(r)=0$.

(2) If $f(0)=0$ and $0<\lim _{r \rightarrow 0^{+}} \frac{f(r)}{r^{\beta}}=A<+\infty$, then

(2a) If $0<\beta<1$, then $\lim _{r \rightarrow 0^{+}} T(r)=0$;

(2b) If $\beta=1$, then $\lim _{r \rightarrow 0^{+}} T(r)=\frac{\pi}{2 \sqrt{\lambda A}}$;

(2c) If $\beta>1$, then $\lim _{r \rightarrow 0^{+}} T(r)=+\infty$.

Lemma 2.4.[13] Assume that $\eta \equiv \lim _{r \rightarrow 0^{+}} \frac{F(r)}{r^{2}}=\lim _{r \rightarrow 0^{+}} \frac{f(r)}{2 r} \in(0, \infty)$, then for $\lambda>0$,

$$
\lim _{r \rightarrow 0^{+}} T^{\prime}(r)= \begin{cases}-\frac{1}{6 \sqrt{2} \eta^{\frac{3}{2}} \sqrt{\lambda}} \lim _{r \rightarrow 0^{+}} f^{\prime \prime}(r), & \text { if } \lim _{r \rightarrow 0^{+}} f^{\prime \prime}(r) \text { exists } \\ -\infty, & \text { if } \lim _{r \rightarrow 0^{+}} f^{\prime \prime}(r)=\infty\end{cases}
$$

Lemma 2.5.[5] Assume that (H1) holds and $0<\lim _{r \rightarrow 0^{+}} \frac{f(r)}{r^{\beta}}=A<+\infty$, then

(1) If $0<\beta<1$, then $\lim _{r \rightarrow 0^{+}} T^{\prime}(r)=+\infty$.

(2) If $\beta>1$, then $\lim _{r \rightarrow 0^{+}} T^{\prime}(r)=-\infty$.

Lemma 2.6. Assume that $f$ satisfies (H1) and (H3), then for any $\lambda>0$,

$$
T^{\prime \prime}(r)>0, \quad r \in(0, p) .
$$

Proof It is easy to know that $\Delta F(2+\Delta F)>0$ and $1+\Delta F>\frac{1}{2}(2+\Delta F)$, then

$$
\begin{aligned}
T^{\prime \prime}(r) & =\frac{1}{r^{2}} \int_{0}^{r} \frac{3 \Delta \bar{f}^{2}(1+\Delta F)-\Delta F(2+\Delta F)\left(2 \Delta \bar{f}+\Delta \bar{f}^{\prime}\right)}{(\Delta F(2+\Delta F))^{\frac{5}{2}}} d u \\
& >\frac{1}{2 r^{2}} \int_{0}^{r} \frac{(2+\Delta F)\left(3 \Delta \bar{f}^{2}-4 \Delta F \Delta \bar{f}-2 \Delta F \Delta \bar{f}^{\prime}\right)}{(\Delta F(2+\Delta F))^{\frac{5}{2}}} d u \\
& =\frac{1}{2 r^{2}} \int_{0}^{r} \frac{(2+\Delta F)\left(3 \Delta \bar{f}(\Delta \bar{f}-2 \Delta F)+2 \Delta F \Delta \bar{f}-2 \Delta F \Delta \bar{f}^{\prime}\right)}{(\Delta F(2+\Delta F))^{\frac{5}{2}}} d u .
\end{aligned}
$$


Let $g_{1}(u)=u f(u)-2 F(u)$. From Lemma 2.1, $\Delta \bar{f}-2 \Delta F=\lambda\left(g_{1}(r)-g_{1}(u)\right), u \in(0, r)$. It follows from (H3) that $g_{1}^{\prime}(u)=u f^{\prime}(u)-f(u) \geq 0, u \in(0, p)$, which implies that, for any $r \in(0, p)$

$$
\Delta \bar{f}-2 \Delta F \geq 0, u \in(0, r)
$$

According to (2.4), we get

$$
\begin{aligned}
3 \Delta \bar{f}(\Delta \bar{f}-2 \Delta F)+2 \Delta F \Delta \bar{f}-2 \Delta F \Delta \bar{f}^{\prime} & \geq 6 \Delta F(\Delta \bar{f}-2 \Delta F)+2 \Delta F \Delta \bar{f}-2 \Delta F \Delta \bar{f}^{\prime} \\
& =2 \Delta F\left(4 \Delta \bar{f}-6 \Delta F-\Delta \bar{f}^{\prime}\right) .
\end{aligned}
$$

Let $g_{2}(u)=4 u f(u)-6 F(u)-u^{2} f^{\prime \prime}(u)$. From Lemma 2.1, $4 \Delta \bar{f}-6 \Delta F-\Delta \bar{f}^{\prime}=\lambda\left(g_{2}(r)-g_{2}(u)\right)$, $u \in(0, r)$. According to (H3), we obtain that $g_{2}^{\prime}(u)=2 u f^{\prime}(u)-2 f(u)-u^{2} f^{\prime \prime}(u) \geq 0, u \in(0, p)$, which implies that, for any $r \in(0, p)$,

$$
4 \Delta \bar{f}-6 \Delta F-\Delta \bar{f}^{\prime} \geq 0, u \in(0, r) .
$$

Therefore, $T^{\prime \prime}(r)>0, r \in(0, p)$.

Lemma 2.7. Assume that $f$ satisfies (H1), (H2) and (H3), then for any $\lambda>0$,

$$
T^{\prime \prime}(r)+\frac{2}{r} T^{\prime}(r)>0, \quad r \in[p, L) .
$$

Proof Let $\theta_{\lambda}(u)=2 F_{\lambda}(u)-u f_{\lambda}(u)$, then

$$
\begin{gathered}
\theta_{\lambda}^{\prime}(u)=f_{\lambda}(u)-u f_{\lambda}^{\prime}(u), \\
\theta_{\lambda}^{\prime \prime}(u)=-u f_{\lambda}^{\prime \prime}(u) \begin{cases}<0, & \text { on }(0, \gamma), \\
=0, & \text { when } u=\gamma . \\
>0, & \text { on }(\gamma, L) .\end{cases}
\end{gathered}
$$

Since $f(0)=0, \theta_{\lambda}^{\prime}(0)=0$, the curves of $\theta_{\lambda}(u)$ and $\theta_{\lambda}^{\prime}(u)$ can be depicted in Fig.2.

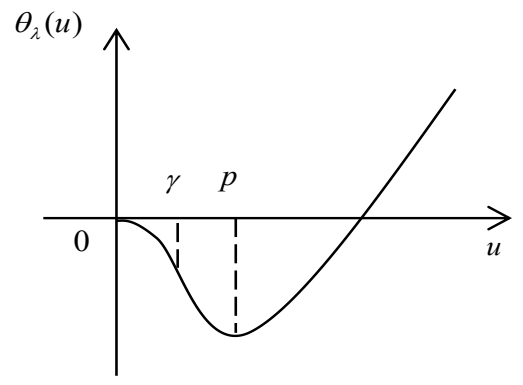

(a)

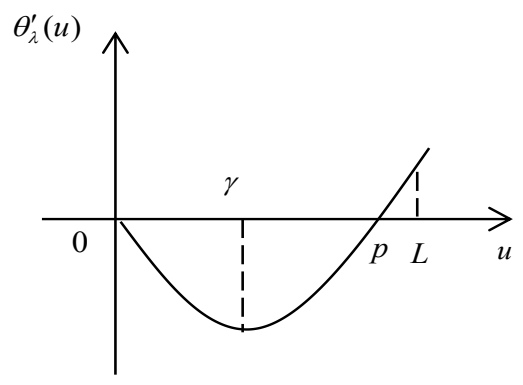

(b)

Fig. 2. (a) Graph of $\theta_{\lambda}(u)$; (b) Graph of $\theta_{\lambda}^{\prime}(u)$. 
Let $\phi_{\lambda}(u)=u \theta_{\lambda}^{\prime}(u)-\theta_{\lambda}(u)$, then

$$
\phi_{\lambda}^{\prime}(u)=u \phi_{\lambda}^{\prime \prime}(u)=-u^{2} f_{\lambda}^{\prime \prime}(u) \begin{cases}<0, & \text { on }(0, \gamma), \\ =0, & \text { when } u=\gamma \\ >0, & \text { on }(\gamma, L),\end{cases}
$$

and $\phi_{\lambda}(p)=-\theta_{\lambda}(p)>0$. From $\theta_{\lambda}(0)=0, \phi_{\lambda}(0)=0$, the graph of $\phi_{\lambda}(u)$ can be depicted in Fig.3.

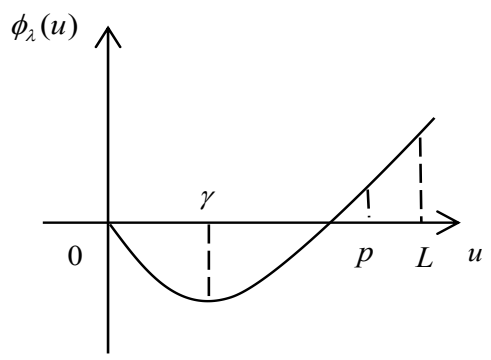

Fig. 3. Graph of $\phi_{\lambda}(u)$.

By Lemma 2.1, we have

$$
\begin{gathered}
2 \Delta F-\Delta \bar{f}=2 F_{\lambda}(r)-r f_{\lambda}(r)-2 F_{\lambda}(u)+u f_{\lambda}(u)=\theta_{\lambda}(r)-\theta_{\lambda}(u), \\
2 \Delta \bar{f}-2 \Delta F-\Delta \bar{f}^{\prime}=r \theta_{\lambda}^{\prime}(r)-\theta_{\lambda}(r)-u \theta_{\lambda}^{\prime}(u)+\theta_{\lambda}(u)=\phi_{\lambda}(r)-\phi_{\lambda}(r) .
\end{gathered}
$$

As $\Delta F(2+\Delta F)>0$ and $1+\Delta F>\frac{1}{2}(2+\Delta F)$,

$$
\begin{aligned}
T^{\prime \prime}(r)+\frac{2}{r} T^{\prime}(r) & =\frac{1}{r^{2}} \int_{0}^{r} \frac{3 \Delta \bar{f}^{2}(1+\Delta F)-\Delta F(2+\Delta F)\left(2 \Delta \bar{f}+\Delta \bar{f}^{\prime}\right)+2 \Delta F(2+\Delta F)[\Delta F(2+\Delta F)(1+\Delta F)-\Delta \bar{f}]}{(\Delta F(2+\Delta F))^{\frac{5}{2}}} d u \\
& >\frac{1}{2 r^{2}} \int_{0}^{r} \frac{(2+\Delta F)\left(3 \Delta \bar{f}^{2}-8 \Delta F \Delta \bar{f}-2 \Delta F \Delta \bar{f}^{\prime}+8 \Delta F^{2}\right)}{(\Delta F(2+\Delta F))^{\frac{5}{2}}} d u \\
& =\frac{1}{2 r^{2}} \int_{0}^{r} \frac{(2+\Delta F)\left(3\left(\theta_{\lambda}(r)-\theta_{\lambda}(u)\right)^{2}+2 \Delta F\left(\phi_{\lambda}(r)-\phi_{\lambda}(u)\right)\right)}{(\Delta F(2+\Delta F))^{\frac{5}{2}}} d u \\
& >\frac{1}{2 r^{2}} \int_{0}^{r} \frac{\phi_{\lambda}(r)-\phi_{\lambda}(u)}{(\Delta F(2+\Delta F))^{\frac{3}{2}}} d u .
\end{aligned}
$$

For $r \in[p, L)$, we know that $\phi_{\lambda}(r)-\phi_{\lambda}(u)>0, u \in(0, r)$. Therefore,

$$
T^{\prime \prime}(r)+\frac{2}{r} T^{\prime}(r)>0, r \in[p, L) .
$$

\section{Proof of the main results}

Proof of Theorem 1.1. According to the definition of time map and $\left|u^{\prime}(x)\right|<1$, positive solutions of problem (1.1) is equivalent to finding $r \in(0, L)$ such that

$$
T(r)=L
$$


Therefore, the positive solutions of problem (1.1) correspond to the number of solutions of (3.1).

As $\lim _{r \rightarrow 0^{+}} \frac{f(r)}{r}=A \in(0, \infty)$, by Lemma 2.3, we have $\lim _{r \rightarrow 0^{+}} T(r)=\frac{\pi}{2 \sqrt{\lambda A}}$. From $\frac{\pi}{2 \sqrt{\lambda A}}=L$, $\lambda=\frac{\pi^{2}}{4 L^{2} A} \triangleq \lambda^{*}$. By calculation,

$$
\lim _{r \rightarrow L^{-}} T(r)=\lim _{r \rightarrow L^{-}} r \int_{0}^{1} \frac{1+\lambda(F(r)-F(r s))}{\sqrt{\lambda(F(r)-F(r s))(2+\lambda(F(r)-F(r s)))}} d s=L \quad \text { as } \quad \lambda \rightarrow \infty .
$$

By Lemma 2.4, $\lim _{r \rightarrow 0^{+}} T^{\prime}(r)<0$. Moreover, Lemma 2.6 and Lemma 2.7 imply that $T(r)$ has exactly one critical point for $r \in(0, L)$. Therefore, combining with Lemma 2.2, the graph of $T(r)$ for $\lambda \geq \lambda_{*}$ can be depicted in Fig.4(a).

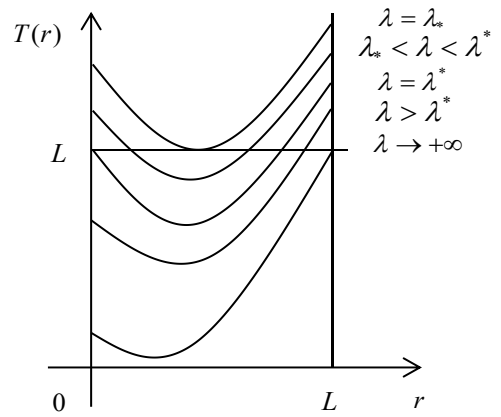

(a)

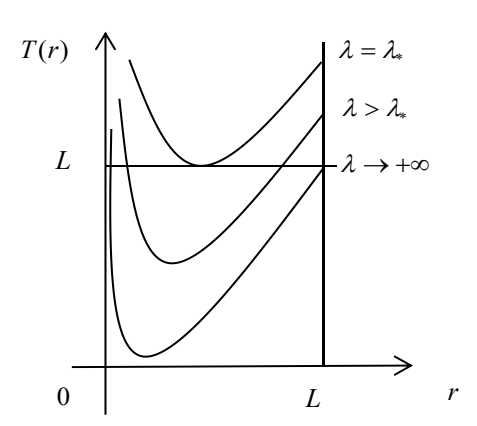

(b)

Fig. 4. (a) Graph of $T(r)$ in Theorem 1.1; (b) Graph of $T(r)$ in Theorem 1.2.

There exists $0<\lambda_{*}<\lambda^{*}=\frac{\pi^{2}}{4 L^{2} A}$ such that problem (1.1) has zero, exactly one or exactly two positive solutions according to $\lambda \in\left(0, \lambda_{*}\right), \lambda=\lambda_{*}$ and $\lambda \geq \lambda^{*}, \lambda \in\left(\lambda_{*}, \lambda^{*}\right)$ respectively.

Proof of Theorem 1.2. According to the definition of time map and $\left|u^{\prime}(x)\right|<1$, positive solutions of problem (1.1) is equivalent to finding $r \in(0, L)$ such that

$$
T(r)=L
$$

Therefore, the positive solutions of problem (1.1) correspond to the number of solutions of (3.2).

By Lemma 2.3 and Lemma 2.5, we obtain $\lim _{r \rightarrow 0^{+}} T(r)=+\infty, \lim _{r \rightarrow 0^{+}} T^{\prime}(r)=-\infty$. By calculation, for given $r_{0}>0$,

$$
\lim _{\lambda \rightarrow+\infty} T\left(r_{0}\right)=\lim _{\lambda \rightarrow+\infty} r_{0} \int_{0}^{1} \frac{1+\lambda\left(F\left(r_{0}\right)-F\left(r_{0} s\right)\right)}{\sqrt{\lambda\left(F\left(r_{0}\right)-F\left(r_{0} s\right)\right)\left(2+\lambda\left(F\left(r_{0}\right)-F\left(r_{0} s\right)\right)\right.}} d s=r_{0},
$$

which implies that there exists $r_{0} \in(0, L)$ such that $\lim _{\lambda \rightarrow+\infty} T\left(r_{0}\right)<L$. Moreover, Lemma 2.6 and Lemma 2.7 imply that $T(r)$ has exactly one critical point for $r \in(0, L)$. Therefore, combining with Lemma 2.2, the graph of $T(r)$ for $\lambda \geq \lambda_{*}$ can be depicted in Fig.4(b).

There exists $\lambda_{*}>0$ such that problem (1.1) has zero, exactly one or exactly two positive solutions according to $\lambda \in\left(0, \lambda_{*}\right), \lambda=\lambda_{*}, \lambda \in\left(\lambda_{*},+\infty\right)$ respectively. 


\section{Applications}

Example 4.1 Consider $f(u)=\frac{u(1+u)}{\sqrt{1+u^{2}}}$ for $u>0, L>1$.

By calculation, $f(0)=0, f^{\prime \prime}(u)=\frac{2-3 u-u^{2}}{\left(1+u^{2}\right)^{\frac{5}{2}}}, u f^{\prime}(u)-f(u)-\frac{1}{2} u^{2} f^{\prime \prime}(u)=\frac{u^{3}\left(1+3 u-2 u^{2}\right)}{2\left(1+u^{2}\right)^{\frac{5}{2}}}$ and $u f^{\prime}(u)-f(u)=\frac{u^{2}(1-u)}{\left(1+u^{2}\right)^{\frac{3}{2}}}$, so there exists $\gamma=\frac{\sqrt{17}-3}{2}$ and $p=1$ such that (H2) and (H3) are satisfied. Since $f(u)>0$ for $u>0$, and $\lim _{r \rightarrow 0^{+}} \frac{f(r)}{r}=\lim _{r \rightarrow 0^{+}} \frac{1+r}{\sqrt{1+r^{2}}}=1, \lim _{r \rightarrow 0^{+}} f^{\prime \prime}(r)=2>0,(\mathrm{H} 1)$ and (C1) hold. Therefore, by Theorem 1.1, there exists $0<\lambda_{*}<\lambda^{*}=\frac{\pi^{2}}{4 L^{2}}$ such that problem (1.1) has zero, exactly one or exactly two positive solutions according to $\lambda \in\left(0, \lambda_{*}\right), \lambda=\lambda_{*}$ and $\lambda \geq \lambda^{*}, \lambda \in\left(\lambda_{*}, \lambda^{*}\right)$ respectively.

Example 4.2 Consider $f(u)=\ln \left(1+u^{2}\right)$ for $u>0, L>\frac{2311}{1167}$.

By calculation, $f(0)=0, f^{\prime \prime}(u)=\frac{2\left(1-u^{2}\right)}{\left(1+u^{2}\right)^{2}}, u f^{\prime}(u)-f(u)-\frac{1}{2} u^{2} f^{\prime \prime}(u)=\frac{u^{2}\left(3 u^{2}+1\right)}{\left(1+u^{2}\right)^{2}}$ and $u f^{\prime}(u)-$ $f(u)=\ln \left(1+u^{2}\right)-\frac{2 u^{2}}{\left(1+u^{2}\right)}$, so there exists $\gamma=1$ and $p=\frac{2311}{1167}$ such that (H2) and (H3) are satisfied. Since $f(u)>0$ for $u>0$ and $\lim _{r \rightarrow 0^{+}} \frac{f(r)}{r^{2}}=\lim _{r \rightarrow 0^{+}} \frac{\ln \left(1+r^{2}\right)}{r^{2}}=1$, (H1) and (C2) hold. Therefore, by Theorem 1.2, there exists $\lambda_{*}>0$ such that problem (1.1) has zero, exactly one or exactly two positive solutions according to $\lambda \in\left(0, \lambda_{*}\right), \lambda=\lambda_{*}, \lambda \in\left(\lambda_{*},+\infty\right)$ respectively.

Example 4.3 Consider $f(u)=\frac{u^{2}}{1+u^{2}}$ for $u>0, L>1$.

By calculation, $f(0)=0, f^{\prime \prime}(u)=\frac{2\left(1-3 u^{2}\right)}{\left(1+u^{2}\right)^{3}}, u f^{\prime}(u)-f(u)-\frac{1}{2} u^{2} f^{\prime \prime}(u)=\frac{u^{4}\left(3-u^{2}\right)}{\left(1+u^{2}\right)^{3}}$ and $u f^{\prime}(u)-f(u)=\frac{u^{2}\left(1-u^{2}\right)}{\left(1+u^{2}\right)^{2}}$, so there exists $\gamma=\frac{1}{\sqrt{3}}$ and $p=1$ such that condition (H2) and (H3) are satisfied. Since $f(u)>0$ for $u>0$ and $\lim _{r \rightarrow 0^{+}} \frac{f(r)}{r^{2}}=\lim _{r \rightarrow 0^{+}} \frac{1}{1+r^{2}}=1$, (H1) and (C2) hold. Therefore, by Theorem 1.2, there exists $\lambda_{*}>0$ such that problem (1.1) has zero, exactly one or exactly two positive solutions according to $\lambda \in\left(0, \lambda_{*}\right), \lambda=\lambda_{*}, \lambda \in\left(\lambda_{*},+\infty\right)$ respectively.

\section{Availability of data and materials}

Not applicable.

\section{Competing interests}

The authors declare that they have no competing interests regarding the publication of this paper.

\section{Funding}

This work was supported by the National Natural Science Foundation of China (No.11801243 and No.11961039), the Young Scholars Science Foundation of Lanzhou Jiaotong University 
(No.2017012).

\section{Authors' contributions}

All authors completed the main study, carried out the results of this article and drafted the paper. The first author checked the proofs and verified the calculation. All authors read and approved the final manuscript.

\section{Acknowledgements}

Not applicable.

\section{References}

[1] C. Bereanu, P. Jebelean, P. J. Torres, Positive radial solutions for Dirichlet problems with mean curvature operators in Minkowski space, J. Funct. Anal. 264 (2013), no. 1, 270-287.

[2] C. Bereanu, P. Jebelean, P. J. Torres, Multiple positive radial solutions for a Dirichlet problem involving the mean curvature operator in Minkowski space, J. Funct. Anal. 265 (2013), no. 4, 644-659.

[3] G. Dai, Bifurcation and positive solutions for problem with mean curvature operator in Minkowski space, Calc. Var. Partial Diff. Equ. 55 (2016), no. 5, 1-17.

[4] G.Dai, Global bifurcation for problem with mean curvature operator on general domain, Nonlinear Differential Equations Appl. 3 (2017), no. 24, 1-10.

[5] H. Gao, J. Xu, Bifurcation curves and exact multiplicity of positive solutions for Dirichlet problems with the Minkowski-curvature equation, Bound. Value Probl. (2021) 2021:81.

[6] I. Coelho, C. Corsato, F. Obersnel, Positive solutions of the Dirichlet problem for the onedimensional Minkowski-curvature equation, Adv. Nonlinear Stud. 12 (2012), no.3, 621-638.

[7] I. Coelho, C. Corsato, S. Rivetti, Positive radial solutions of the Dirichlet problem for the Minkowski-curvature equation in a ball, Topol. Methods Nonlinear. Anal. 44 (2014), no. 1, $23-39$. 
[8] K.-C. Hung, S.-H. Wang, A theorem on S-shaped bifurcation curve for a positone problem with convex-concave nonlinearity and its applications to the perturbed Gelfand problem, J. Differential Equations 251 (2011), no. 2, 223-237.

[9] P. Korman, Y. Li, On the exactness of an S-shaped bifurcation curve, Proc. Amer. Math. Soc. 127 (1999), no. 4, $1011-1020$.

[10] P. Korman, Y. Li, Exact multiplicity of positive solutions for concave - convex and convex - concave nonlinearities, J. Differential Equations 257 (2014), , no. 10, 3730-3737.

[11] R. Ma, H. Gao, Y. Lu, Global structure of radial positive solutions for a prescribed mean curvature problem in a ball, J. Funct. Anal. 270 (2016), no. 7, 2430-2455.

[12] R. Ma, T. Chen, H. Gao, On positive solutions of the Dirichlet problem involving the extrinsic mean curvature operator, Electron. J. Qual. Theory Differ. Equ. 2016, no. 98, $1-10$.

[13] S.-Y. Huang, Exact multiplicity and bifurcation curves of positive solutions of a onedimensional Minkowski-curvature problem and its application, Commun. Pur. Appl. Anal. 17 (2018), no. 3, 1271-1294.

[14] S.-Y. Huang, Classification and evolution of bifurcation curves for the one-dimensional Minkowski-curvature problem and its applications, J. Differential Equations 264 (2018), no. $9,5977-6011$.

[15] X. Zhang, M. Feng, Bifurcation diagrams and exact multiplicity of positive solutions of onedimensional prescribed mean curvature equation in Minkowski space, Commun. Contemp. Math. 21 (2019), no. 3, 1-15. 